Z Gerontol Geriat 2013 · 46:304-305

DOI 10.1007/s00391-013-0499-3

Online publiziert: 4. Mai 2013

๑) Springer-Verlag Berlin Heidelberg 2013

C. Kricheldorff' · C. Tesch-Römer ${ }^{2}$

${ }^{1}$ Katholische Hochschule Freiburg

${ }^{2}$ Deutsches Zentrum für Altersfragen (DZA), Berlin

\title{
Altern und soziale Ungleichheit
}

Der Fachdiskurs über soziale Ungleichheit im Alter war in den vergangenen Jahrzehnten von einem mehrfachen Paradigmenwechsel geprägt. Nachdem das Phänomen der Altersarmut und der damit verbundenen sozialen Exklusion, das in den 1960er und 1970er Jahren vor allem alleinstehende alte Frauen mit geringen Rentenansprüchen betraf, weitgehend gesellschaftlich überwunden schien, wurde der Blick ab Anfang der 1990er Jahre verstärkt auf die gut gebildeten und materiell überdurchschnittlich ausgestatteten „neuen Alten“ gerichtet. Deren Ressourcen und Potenziale standen, vor dem Hintergrund sich verändernder gesellschaftlicher Bedingungen im demographischen Wandel, lange Zeit so im Mittelpunkt der gerontologischen Forschung, dass darüber die eher benachteiligten Älteren eher aus dem Blick gerieten. Auch in der öffentlichen und medialen Wahrnehmung wurde die Lebensphase Alter mit einem privilegierten Status hinsichtlich der materiellen und sozialen Sicherung konnotiert.

In Deutschland erhielt ab Mitte der 2000er Jahre die Diskussion über eine künftig wieder wachsende Relevanz von Altersarmut eine neue Brisanz. Vor allem über die Prognosen bezüglich einer mangelhaften materiellen Absicherung durch die gesetzlichen Renten für künftige Alterskohorten erhält der aktuelle fachliche Diskurs über soziale Ungleichheit in der Traditionslinie der kritischen Gerontologie eine wachsende Bedeutung. Es werden Szenarien vorgezeichnet, nach denen die zunehmende Abhängigkeit von Sozialleistungen wieder typischer für die Lebensphase Alter werden könnte und Armut im
Rentenalter für jeden dritten Arbeitnehmer (35\% der sozialversicherungspflichtigen Vollzeitbeschäftigten) als wahrscheinlich beschrieben wird.

In diesem Heft geht es um Analysen zur Heterogenität des Alters, zur Verschiedenartigkeit von Lebenslagen und zu Disparitäten im Lebenslauf, die sich im Alter in kumulativer Form zeigen. Dabei sind verschiedene Perspektiven zu unterscheiden. Zunächst einmal kann, einer materiellen Ressourcenlogik folgend, die materielle Lebenssituation in den Blick genommen werden. Soziale Ungleichheit im Alter ist in dieser Perspektive die ungleiche Verteilung von Einkommen und Vermögen. In einer zweiten Perspektive können die lebenslangen Folgen sozialer Ungleichheit für die Lebenssituation älter werdender und alter Menschen betrachtet werden. Hier wird neben der materiellen Lebensgrundlage häufig auch Bildung und sozialer Status berücksichtigt. Biographisch über den Lebenslauf wirksame soziale Ungleichheit hat einen erheblichen Einfluss auf das Ausmaß sozialer Einbindung und damit auch auf die Chancen gesellschaftlicher Teilhabe. Über den Zusammenhang von sozialer Ungleichheit und Gesundheit liegen reichhaltige empirische Daten und $\mathrm{Be}$ funde vor. Angesichts wachsender Disparitäten im Alter sind Maßnahmen zur Förderung der sozialen Integration benachteiligter Gruppen Älterer notwendig. Alterspolitik sollte daher nicht allein die Reduzierung materieller Ungleichheit im Alter in den Blick nehmen, sondern zusätzlich auch Rahmenbedingungen für eine soziale Altenarbeit schaffen, 
die auf Inklusion und Förderung sozialer Teilhabe älterer Menschen gerichtet ist.

\section{》) Die soziale Integration benachteiligter Älterer muss gefördert werden}

Soziale Ungleichheit im Alter entsteht aber auch, weil die Lebensphase "Alter" gegenüber anderen Lebensphasen diskriminiert wird. Altersdiskriminierung findet auf der strukturellen und institutionellen Ebene statt und sie zeigt sich als symbolische und sprachliche Diskriminierung. Da ist die Rede von der Rentnerschwemme, von der Alterslast oder vom Pflegenotstand, den die Alten verursachen. Über die Wirkungen von einer solchen Art symbolischer Diskriminierung ist bekannt, dass sie sich in der Folge in einem geringen Selbstbewusstsein der Betroffenen niederschlagen kann, was zu resignativen Haltungen führt. Es sollte zu denken geben, dass sich ältere und alte Menschen offenkundig nicht zu erlebten Diskriminierungen äußern und für sich kaum Möglichkeiten sehen, dagegen aktiv vorzugehen. Darauf weist eine Erhebung hin, die von der Bundesarbeitsgemeinschaft der Seniorenorganisationen (BAGSO) und dem Kuratorium Deutsche Altershilfe (KDA) im Jahr 2012 veröffentlicht wurde. Dieses Phänomen birgt in sich die Gefahr der Selbststigmatisierung alter Menschen, die zur Selbstausgrenzung und damit in der Konsequenz zu sozialer Ungleichheit führen kann.

Im Themenschwerpunkt des vorliegenden Hefts wird Altern und soziale Ungleichheit aus verschiedenen Perspektiven beleuchtet. Ein erster Beitrag stellt Ergebnisse aus einem SILQUA-Projekt vor (Förderschwerpunkt "Soziale Innovationen Lebensqualität im Alter" des BMBF) und nimmt alternde Stadtgesellschaften in den Blick. Dabei werden auch Strategien und Wege aufgezeigt, älteren Menschen mit niedrigen Bildungsabschlüssen und geringem Einkommen den Weg zu sozialer und politischer Partizipation zu ermöglichen. Auf der Basis der Ergebnisse des Deutschen Altersurveys (DEAS) wird aufgezeigt, auf welche Weise bürgerschaftliche Beteiligung und Partizipation mit den entscheidenden Einflussfaktoren auf soziale Ungleichheit - Einkommen, Bildung und Status - korrelieren (leider kann dieser Beitrag erst in Heft 5 der ZGG erscheinen). Soziale Ungleichheit in der ambulanten Pflege wird in einem dritten Beitrag thematisiert. Dabei steht die Frage im Mittelpunkt, ob und in welcher Form Pflegekräfte in ambulanten Pflegediensten soziale Ungleichheit in ihrem Arbeitsalltag feststellen und wie ihre Arbeitssituation davon beeinflusst wird. Ein vierter Beitrag stellt die Ergebnisse einer qualitativen Studie vor, die die soziale Situation von alten Menschen im Strafvollzug untersucht hat, durchgeführt in der Justizvollzugsanstalt Berlin-Tegel.

In allen Beiträgen wird deutlich, dass Alter und Altern mit vielen Formen sozialer Ungleichheit in Verbindung gebracht werden können und müssen. Die gerontologische Forschung hat viele Aspekte bislang noch wenig im Blick, Forschungsdesiderate werden deutlich. Der Themenschwerpunkt versteht sich vor diesem Hintergrund als Anstoß und Ermutigung, in der Tradition der kritischen Gerontologie bislang vernachlässigte Themenfelder aufzugreifen.
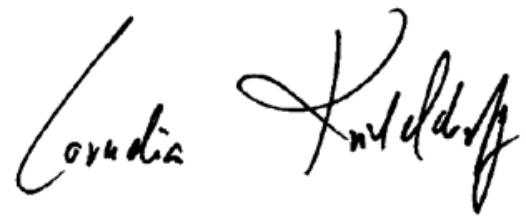

C. Kricheldorff

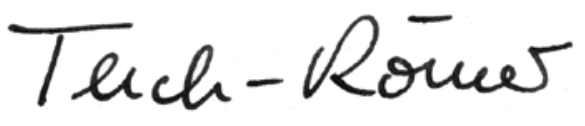

C. Tesch-Römer

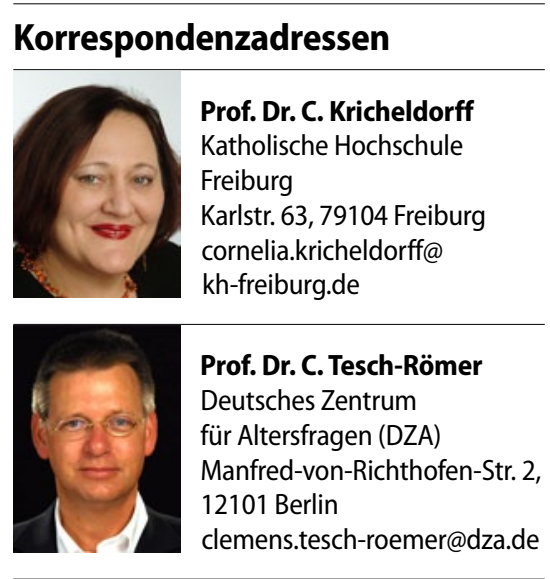

